Bull. Austral. Math. Soc.

$52 \mathrm{~A} 20,52 \mathrm{~A} 41$

VoL. 53 (1996) [159-167]

\title{
DEGREE OF POINTEDNESS OF A CONVEX FUNCTION
}

\section{Alberto SeEger}

A convex function $f$ is said to be pointed if its epigraph has a recession cone which is pointed. Partial pointedness of $f$ refers to the case in which such a recession cone is only partially pointed. In this note we show that the degree of pointedness of $f$ is related to the "thickness" of the effective domain of the conjugate function $f^{*}$.

\section{INTRODUCTION}

Let $K$ be a closed convex cone in some finite dimensional linear space $X$. It is easy to see that

$$
\ell(K):=K \cap-K
$$

is the largest subspace of $X$ which is contained in $K$. The dimension of such a subspace can be used to measure the degree of pointedness of $K$.

Definition 1: The degree of pointedness of $K$ is defined as the integer

$$
p[K]:=\operatorname{dim} X-\operatorname{dim} \ell(K) .
$$

If $p[K]=\operatorname{dim} X$, then one says that $K$ is pointed. If $0<p[K]<\operatorname{dim} X$, then one says that $K$ is partially pointed.

Consider a function $f: X \rightarrow R \cup\{+\infty\}$ whose effective domain

$$
\operatorname{dom} f:=\{x \in X: f(x)<+\infty\}
$$

is nonempty, and whose epigraph

$$
\text { epi } f:=\{(x, \lambda) \in X \times R: f(x) \leqslant \lambda\}
$$

is convex and closed. The class of such functions is usually denoted by $\Gamma_{0}(X)$. The recession cone of the set epi $f$ is defined by

$$
(\text { epi } f)_{\infty}:=\{(u, \alpha) \in X \times R: \quad(u, \alpha)+\text { epi } f \subseteq \text { epi } f\} .
$$

Received 15th May, 1995

Copyright Clearance Centre, Inc. Serial-fee code: 0004-9729/96 \$A2.00+0.00. 
The recession function $f_{\infty}$ of $f$ is given by

$$
f_{\infty}(u):=\sup \{f(x+u)-f(x): x \in \operatorname{dom} f\} \quad \text { for all } u \in X .
$$

Both notions are standard in the context of convex analysis and can be consulted, for instance, in the book by Rockafellar [3]. In this note we study the following new concept.

Definition 2: The degree of pointedness of the function $f \in \Gamma_{0}(X)$ is the integer

$$
p[f]:=\operatorname{dim} X-\operatorname{dim} \ell\left((\text { epi } f)_{\infty}\right) .
$$

If $p[f]=\operatorname{dim} X$, then $f$ is said to be pointed. If $0<p[f]<\operatorname{dim} X$, then $f$ is called partially pointed.

REMARK. According to the above definition, $f$ is pointed if and only if (epi $f)_{\infty}$ is a pointed cone. This case has already been considered by Benoist and Hiriart-Urruty [1, Definition 2.3]. However, these authors do not address the question of the dimension of $\ell\left((\text { epi } f)_{\infty}\right)$.

\section{Pointedness and Conjugacy.}

In this section we derive a simple formula for computing the degree of pointedness of a function $f \in \Gamma_{0}(X)$. Recall that the Legendre-Fenchel conjugate of $f$ is the function $f^{*} \in \Gamma_{0}(X)$ defined by

$$
f^{*}(y):=\sup _{x \in X}\{\langle y, x\rangle-f(x)\} \quad \text { for all } y \in X,
$$

where $\langle\cdot, \cdot\rangle$ is a given inner product in the space $X$. The next theorem says that the degree of pointedness of $f$ is equal to the dimension of the effective domain of $f^{*}$. The dimension of a nonempty convex set $A \subseteq X$ is defined as the dimension of the affine hull of $A$ (see $[3, \mathrm{p} .12]$ ).

Theorem 1. Let $X$ be a finite dimensional linear space, and let $f \in \Gamma_{0}(X)$. Then,

$$
\operatorname{dim}\left(\operatorname{dom} f^{*}\right)+\operatorname{dim} \ell\left((\text { epi } f)_{\infty}\right)=\operatorname{dim} X
$$

ProOF: We start by proving the equality

$$
\ell\left((\text { epi } f)_{\infty}\right)=\left\langle\operatorname{dom} f^{*} \times\{-1\}\right\rangle^{\perp} .
$$

The notation $\langle C\rangle$ refers to the linear space spanned by the set $C$, and $\langle C\rangle^{\perp}$ stands for the orthogonal complement of $\langle C\rangle$. By definition one has

$$
(u, \alpha) \in \ell\left((\text { epi } f)_{\infty}\right) \Leftrightarrow(u, \alpha) \in(\text { epi } f)_{\infty} \cap-(\text { epi } f)_{\infty} .
$$


Since $(\text { epi } f)_{\infty}=$ epi $f_{\infty}$, one can also write

$$
\begin{aligned}
(u, \alpha) \in \ell\left((\text { epi } f)_{\infty}\right) & \Leftrightarrow f_{\infty}(u) \leqslant \alpha \quad \text { and } f_{\infty}(-u) \leqslant-\alpha \\
& \Leftrightarrow f_{\infty}(u) \leqslant \alpha \leqslant-f_{\infty}(-u) .
\end{aligned}
$$

Now we use the fact that $f_{\infty}$ is the support function of the set $\operatorname{dom} f^{*}$ (see [2]), that is,

$$
f_{\infty}(u)=\sup \left\{\langle y, u\rangle: y \in \operatorname{dom} f^{*}\right\}
$$

One has also

$$
-f_{\infty}(-u)=\inf \left\{\langle y, u\rangle: y \in \operatorname{dom} f^{*}\right\}
$$

Hence

$$
\begin{aligned}
(u, \alpha) \in \ell\left((\text { epi } f)_{\infty}\right) & \Leftrightarrow\langle y, u\rangle=\alpha \quad \text { for all } y \in \operatorname{dom} f^{*} \\
& \Leftrightarrow\langle(y, \beta),(u, \alpha)\rangle=0 \quad \text { for all }(y, \beta) \in \operatorname{dom} f^{*} \times\{-1\} .
\end{aligned}
$$

Equality (4) is proven in this way. To complete the proof of the theorem, it suffices to observe that

$$
\operatorname{dim} A=\operatorname{dim}\langle A \times\{-1\}\rangle-1,
$$

whenever $A$ is a nonempty convex set in $X$. The proof of (5) is essentially an exercise in linear algebra.

Remark. By combining Theorem 1 and [3, Theorem 13.4], one sees that $\ell\left((\text { epi } f)_{\infty}\right)$ has the same dimension as the set $\left\{u \in X: f_{\infty}(u)=-f_{\infty}(-u)\right\}$. The latter set is known as the lineality space of $f$.

We end this section by illustrating Theorem 1 with two examples.

EXAMPLE 1. Consider the space $X=S_{n}$ of symmetric matrices of order $n \times n$ equipped with the usual inner product $\langle x, y\rangle:=\operatorname{trace}(x y)$. The variance of a matrix $x \in S_{n}$ is defined by

$$
(\operatorname{var})(x):=\frac{1}{n}\langle x, x\rangle-\left(\frac{\operatorname{trace} x}{n}\right)^{2} .
$$

The function var : $S_{n} \rightarrow R$ is convex, and its conjugate (var)* $S_{n} \rightarrow R \cup\{+\infty\}$ is given by

$$
(\operatorname{var})^{*}(y)= \begin{cases}\frac{n}{4}\langle y, y\rangle & \text { if trace } y=0 \\ +\infty & \text { otherwise. }\end{cases}
$$

The dimension of $\operatorname{dom}(\mathrm{var})^{*}=\left\{y \in S_{n}\right.$ : trace $\left.y=0\right\}$ is equal to $\operatorname{dim} S_{n}-1$. Hence, the function var is partially pointed and its degree of pointedness is

$$
p[\operatorname{var}]=\operatorname{dim} S_{n}-1=\left(n^{2}+n-2\right) / 2 .
$$


EXAMPLE 2. Let $X=S_{n}$ be as in Example 1. Let $\lambda_{\max }(x)$ denote the largest eigenvalue of the matrix $x \in S_{n}$. It is known that $\lambda_{\max }: S_{n} \rightarrow R$ is equal to the support function of the set

$$
A=\left\{x \in S_{n}: \quad x \text { is positive semidefinite, } \operatorname{trace} x=1\right\} .
$$

Hence $\operatorname{dom}\left(\lambda_{\max }\right)^{*}=A$ is a set of dimension equal to $\operatorname{dim} S_{n}-1$. Thus, $\lambda_{\max }$ is partially pointed and $p\left[\lambda_{\max }\right]=\left(n^{2}+n-2\right) / 2$. In this example one can also compute $p\left[\lambda_{\max }\right]$ by using the very definition of this number. The set epi $\left(\lambda_{\max }\right)$ is a closed convex cone, so it coincides with its recession cone. One has

$$
\begin{aligned}
(x, \lambda) \in \ell\left(\operatorname{epi}\left(\lambda_{\max }\right)\right) & \Leftrightarrow \lambda_{\max }(x) \leqslant \lambda \text { and } \lambda_{\max }(-x) \leqslant-\lambda \\
& \Leftrightarrow \text { all the eigenvalues of } x \text { are equal to } \lambda .
\end{aligned}
$$

If $i \in S_{n}$ denotes the identity matrix, then

$$
\ell\left(\operatorname{epi}\left(\lambda_{\max }\right)\right)=\{(\lambda i, \lambda): \lambda \in R\}=R\{(i, 1)\} \subset S_{n} \times R
$$

is a space of dimension 1 .

\section{Pointedness and Growth Condition}

Recall that each $f \in \Gamma_{0}(X)$ can be minorised by some affine function, that is, one can find $y \in X$ and $b \in R$ such that

$$
f(x) \geqslant\langle y, x\rangle+b \quad \text { for all } x \in X .
$$

Is it possible to obtain a more precise information on the growth of $f$ ? In other words, is it possible to write a stronger growth condition for $f$ ? As already observed by Benoist and Hiriart-Urruty [1, Theorem 2.4], a growth condition of the type

$$
f(x) \geqslant r\|x\|+\langle y, x\rangle+b \text { for all } x \in X,
$$

with $r>0$, characterises the class of functions $f \in \Gamma_{0}(X)$ which are pointed. This observation leads us to think that a partially pointed function $f \in \Gamma_{0}(X)$ satisfies a growth condition which is intermediate between (6) and (7). The purpose of the next theorem is to display in a clear-cut manner the relationship between growth conditions and degree of pointedness. To start with, observe that the conditions (6) and (7) can be written in a common format, namely:

$$
f(x) \geqslant \psi_{A}^{*}(x)+b \quad \text { for all } x \in X,
$$

where $\psi_{A}^{*}$ denotes the support function of $A \subseteq X$. In the former case $A$ corresponds to the zero-dimensional set $\{y\}$, and in the latter case $A$ is the full dimensional ball $B(y, r):=\{z \in X:\|z-y\| \leqslant r\}$. 
THEOREM 2. The degree of pointedness of the function $f \in \Gamma_{0}(X)$ is given by

$$
p[f]=\max _{A \in C(X)}\left\{\operatorname{dim} A: f-\psi_{A}^{*} \text { is minorised }\right\},
$$

where $C(X)$ denotes the class of nonempty convex sets in $X$. One can also write

$$
p[f]=\max _{A \in C(X)}\left\{\operatorname{dim} A: f_{\infty} \geqslant \psi_{A}^{*}\right\},
$$

where the maximum in (9) is attained at $A=\operatorname{dom} f^{*}$ (or, more generally, at any set $A$ which is contained in the closure of $\operatorname{dom} f^{*}$ and which has the same dimension as $\operatorname{dom} f^{*}$ ).

Proof: The inequality $f_{\infty} \geqslant \psi_{A}^{*}$ is equivalent to the inclusion

$$
\bar{A} \subseteq \overline{\operatorname{dom} f^{*}},
$$

where the upper bar denotes the closure operation in $X$. Since the closure operation does not affect the dimension of a convex set (see [3, Theorem 6.2]), the maximum in (9) is attained at any $A \subseteq \overline{\operatorname{dom} f^{*}}$ which has the same dimension as $\operatorname{dom} f^{*}$. Now we prove that $p[f]=m[f]$, where $m[f]$ denotes the term on the right-hand side of $(8)$. It is fairly clear that

$$
f-\psi_{A}^{*} \text { is minorised } \Rightarrow f_{\infty} \geqslant \psi_{A}^{*} .
$$

Thus (9) yields the inequality $p[f] \geqslant m[f]$. According to the Toland-Singer duality theorem (see $[4,5]$ ), for all $A \in C(X)$, one has:

$$
\inf _{x \in X}\left\{f(x)-\psi_{A}^{*}(x)\right\}=-\sup _{y \in \bar{A}} f^{*}(y) .
$$

Hence,

$$
m[f]=\max _{A \in C(X)}\left\{\operatorname{dim} A: f^{*} \text { is majorised over } \bar{A}\right\} .
$$

Take any $y \in r i\left(\operatorname{dom} f^{*}\right)$, where " $r$ " stands for the relative interior (see $\left.[3, p .44]\right)$. Then, for some $r>0$ sufficiently small, one has

$$
A_{r}:=B(y, r) \cap \overline{\operatorname{dom} f^{*}} \subset r i\left(\operatorname{dom} f^{*}\right) \text {. }
$$

According to Rockafellar [3, Theorem 10.1], the function $f^{*}$ is continuous relative to $r i\left(\operatorname{dom} f^{*}\right)$. Hence, $f^{*}$ is majorised over the compact set $A_{r}$, and

$$
m[f] \geqslant \operatorname{dim} A_{r} .
$$

But

$$
\operatorname{dim} A_{r}=\operatorname{dim}\left(\operatorname{dom} f^{*}\right)=\operatorname{dim}\left(\operatorname{dom} f^{*}\right)=p[f] .
$$

This proves the reverse inequality $m[f] \geqslant p[f]$, and completes the proof of the theorem. 
REMARK. Since $f$ and $\psi_{A}^{*}$ may take the value $+\infty$ at the same time, we have implicitly adopted the rule $(+\infty)-(+\infty)=+\infty$. The maximum in (8) is attained at a set $A$ of the form $A=B(y, r) \cap \overline{\operatorname{dom} f^{*}}$. This means that the formula (8) remains true if one defines $C(X)$ as the class of nonempty convex compact sets in $X$.

\section{Calculation Rules for the Degree of Pointedness.}

Suppose $f \in \Gamma_{0}(X)$ is constructed from other functions, say $f_{1}, \ldots, f_{N}$, whose degrees of pointedness are known, or can be easily computed. In this case it is helpful to have a formula which relates $p[f]$ to the degrees $p\left[f_{1}\right], \ldots, p\left[f_{N}\right]$ of the component functions. The calculation rules recorded in the first two propositions can be proved in a fairly simple way by using Theorem 1 .

Proposition 1. Let $f \in \Gamma_{0}(X)$. Then,

(a) $p[f] \leqslant p[g]$ for all $g \in \Gamma_{0}(X)$ such that $f \leqslant g$;

(b) $p[f+\ell]=p[f]$ for all affine functions $\ell: X \rightarrow R$;

(c) $p[\lambda f]=p[f]$ for all $\lambda>0$;

(d) $p[f(\cdot / \lambda)]=p[f]$ for all $\lambda>0$;

(e) $p\left[f_{c}\right]=p[f]$, where $c \in X$ and $f_{c}(x)=f(x-c)$.

Proposition 2. Let $X=\prod_{k=1}^{N} X_{k}$ be the Cartesian product of the finite dimensional spaces $X_{1}, \ldots, X_{N}$. Suppose

$$
f(x)=f_{1}\left(x_{1}\right)+\cdots+f_{N}\left(x_{N}\right) \quad \text { for all } x \in X
$$

where $f_{k} \in \Gamma_{0}\left(X_{k}\right)$ for all $k=1, \ldots, N$. Then,

$$
p[f]=\sum_{k=1}^{N} p\left[f_{k}\right]
$$

In particular, $f$ is pointed if and only if all the $f_{k}$ 's are pointed.

In the next four propositions we consider some important functional operations arising in the context of convex analysis, namely, pointwise maximum, addition, closed convex hull, and infimal-convolution.

Proposition 3. Let $f_{1}, \ldots, f_{N} \in \Gamma_{0}(X)$ be finite at some common point, and let $f=\max _{1 \leqslant k \leqslant N} f_{k}$. Then,

$$
p[f] \geqslant \max _{1 \leqslant k \leqslant N} p\left[f_{k}\right]
$$


In particular, $f$ is pointed if at least one of the $f_{k}$ 's is pointed.

Proof: Formula (12) follows from Proposition 1(a). Indeed, since each $f_{k} \leqslant f$, one has

$$
p\left[f_{k}\right] \leqslant p[f] \quad \text { for all } k=1, \ldots, N \text {. }
$$

An alternative proof of (12) is based on Theorem 2 and runs as follows. Let $A_{1}, \ldots, A_{N} \in C(X)$ and $b_{1}, \ldots, b_{N} \in R$ be such that $\operatorname{dim} A_{k}=p\left[f_{k}\right]$, and

$$
f_{k}(x) \geqslant \psi_{A_{k}}^{*}(x)+b_{k} \quad \text { for all } x \in X
$$

Then,

$$
f(x) \geqslant \psi_{A}^{*}(x)+b \quad \text { for all } x \in X,
$$

where $b=\min _{1 \leqslant k \leqslant N} b_{k}$ and $A=$ co $\bigcup_{k=1}^{N} A_{k}$ is the convex hull of the sets $A_{1}, \ldots, A_{N}$. Since $f-\psi_{A}^{*}$ is minorised, we have

$$
p[f] \geqslant \operatorname{dim} A \geqslant \max _{1 \leqslant k \leqslant N} \operatorname{dim} A_{k} .
$$

Proposition 4. Let $f_{1}, \ldots, f_{N} \in \Gamma_{0}(X)$ be finite at some common point, and let $f=\sum_{k=1}^{N} f_{k}$. Then

$$
p[f] \geqslant \operatorname{dim}\left(\operatorname{dom} f_{1}^{*}+\cdots+\operatorname{dom} f_{N}^{*}\right) \geqslant \max _{1 \leqslant k \leqslant N} p\left[f_{k}\right]
$$

In particular, $f$ is pointed if at least one of the $f_{k}$ 's is pointed.

Proof: It is known that $f^{*}$ is equal to the lower-semicontinuous hull of the function

$$
y \in X \mapsto h(y):=\inf \left\{f_{1}^{*}\left(y_{1}\right)+\cdots+f_{N}^{*}\left(y_{N}\right): y_{1}+\cdots+y_{N}=y\right\}
$$

Thus,

$$
\operatorname{dom} f^{*} \supseteq \operatorname{dom} h=\operatorname{dom} f_{1}^{*}+\cdots+\operatorname{dom} f_{N}^{*}
$$

and

$$
\operatorname{dim} \operatorname{dom} f^{*} \geqslant \operatorname{dim}\left(\operatorname{dom} f_{1}^{*}+\cdots+\operatorname{dom} f_{N}^{*}\right) \geqslant \max _{1 \leqslant k \leqslant N} \operatorname{dim} \operatorname{dom} f_{k}^{*} .
$$

Theorem 1 completes the proof of (13).

REMARK. If $\bigcap_{k=1}^{N} \mathrm{ri}\left(\operatorname{dom} f_{k}\right)$ is nonempty, then $h$ is lower-semicontinuous, and the first inequality in $(13)$ becomes $p[f]=\operatorname{dim}\left(\operatorname{dom} f_{1}^{*}+\cdots+\operatorname{dom} f_{N}^{*}\right)$. 
Proposition 5. Let $f$ be the closed convex hull of the functions $f_{1}, \ldots, f_{N} \in$ $\Gamma_{0}(X)$. Suppose all the $f_{k}$ 's are minorised by a common affine function. Then

$$
p[f]=\operatorname{dim} \bigcap_{k=1}^{N} \operatorname{dom} f_{k}^{*} \leqslant \min _{1 \leqslant k \leqslant N} p\left[f_{k}\right] .
$$

Proof: Since $f^{*}=\max \left\{f_{k}^{*}: 1 \leqslant k \leqslant N\right\}$, one has

$$
\operatorname{dom} f^{*}=\bigcap_{k=1}^{N} \operatorname{dom} f_{k}^{*}
$$

Formula (14) follows by applying Theorem 1 .

Proposition 6. Let $f$ be the infimal-convolution of the functions $f_{1}, \ldots, f_{N} \in$ $\Gamma_{0}(X)$. Suppose the sets ri $\left(\operatorname{dom} f_{k}\right), k=1, \ldots, N$ have a point in common. Then

$$
p[f]=\operatorname{dim} \bigcap_{k=1}^{N} \operatorname{dom} f_{k}^{*} \leqslant \min _{1 \leqslant k \leqslant N} p\left[f_{k}\right] .
$$

Proof: The proof is the same as in Proposition 4. This time one starts with the equality

$$
(f)^{*}=\sum_{k=1}^{N} f_{k}^{*} .
$$

An important use of the infimal-convolution operation is the regularisation of a given function $f \in \Gamma_{0}(X)$. The regularised version of $f$ is defined by

$$
x \in X \mapsto[f \square \theta](x):=\inf _{u \in X}\{f(u)+\theta(x-u)\}
$$

where the function $\theta \in \Gamma_{0}(X)$ is referred to as a "kernel".

Corollary 1. Let $\theta \in \Gamma_{0}(X)$ be coercive in the sense that $\theta(x) /\|x\| \rightarrow+\infty$ as $\|x\| \rightarrow \infty$. Then

$$
p[f \square \theta]=p[f] \quad \text { for all } f \in \Gamma_{0}(X) .
$$

Proof: That $f \square \theta \in \Gamma_{0}(X)$ follows from the coercivity of $\theta$. One also has $\operatorname{dom} \theta^{*}=X$. Thus, $\operatorname{dom}(f \square \theta)^{*}=\operatorname{dom} f^{*} \cap \operatorname{dom} \theta^{*}$ has the same dimension as $\operatorname{dom} f^{*}$. 


\section{REFERENCES}

[1] J. Benoist and J.B. Hiriart-Urruty, 'Quel est le sous-différentiel de l'enveloppe convexe fermée d'une fonction', C.R. Acad. Sci. Paris. Sér. I 316 (1993), 233-237.

[2] P.J. Laurent, Approximation et optimisation (Hermann, Paris, 1972).

[3] R.T. Rockafellar, Convex analysis (Princeton University Press, Princeton, 1970).

[4] I. Singer, 'A Fenchel-Rockafellar type duality theorem for maximization', Bull. Austral. Math. Soc. 29 (1979), 193-198.

[5] J. Toland, 'A duality principle for nonconvex optimization and the calculus of variations', Arch. Rational Mech. Anal. 71 (1979), 41-61.

King Fahd University of Petroleum and Minerals

Department of Mathematical Sciences

Dhahran 31261

Saudi Arabia 